\title{
Heterosis and Inbreeding Depression for Seed Yield Attributing Traits and Quality Parameter in Cowpea (Vigna unguiculata (L.) Walp.)
}

\author{
R. P. Gupta, S. R. Patel*, Dinisha Abhishek, S.S. Patil and H.N. Patel \\ Department of Genetics \& Plant Breeding, College of Agriculture, Navsari Agricultural \\ University, Campus Bharuch-392 012, India \\ *Corresponding author
}

A B S T R A C T

The present investigation was conducted at College Farm, N. M. College of Agriculture, Navsari Agricultural University, Navsari during kharif - 2016 with a view to study the heterosis and inbreeding depression in five crosses

Keywords

Cow pea, Heterosis, Inbreeding depression and Seed yield

Article Info

Accepted:

26 July 2020

Available Online:

10 August 2020 (each having $\mathrm{P}_{1}, \mathrm{P}_{2}, \mathrm{~F}_{1}, \mathrm{~F}_{2}, \mathrm{BC}_{1}$ and $\mathrm{BC}_{2}$ generations) of cow pea through a compact family block design with three replications. The analysis of variance between crosses revealed that the mean square due to crosses were significant for all the characters except for chlorophyll content. The $\mathrm{F}_{1} \mathrm{~s}$ deviated positively from their batter parent in majority of crosses, indicating importance of heterosis. The magnitude of heterosis were significantly higher for seed yield and its contributing traits i.e. number of pods per plant, number of seeds per pod and 100 seed weight. The crosses which manifested high heterosis for yield and its components also showed high inbreeding depression. Highest heterosis with the lowest inbreeding depression was observed in cross Waghi local $\mathrm{x}$ W-203-1 followed by KM5 x GC-3 and Pant lobia-2 x GC-3, these can further be exploited for commercial point of view.

\section{Introduction}

Pulses are extremely important and cheap source of plant protein (20 to $30 \%$ ) and play a significant role in restoring and enriching soil fertility by fixing atmospheric nitrogen. Legumes contain in their grains nearly about three times the amount of storage proteins found in cereals. The per hectare yield of pulses can be greatly increased following the development and distribution of high yielding hybrids or varieties of these crops.

Due to low productivity of pulses per unit area as compared to cereals, they are less economical to farmers and hence area under 
pulses has slightly decreased (24.0 million hectares in 1961-62 to 23.55 million hectares in 2014-15) (Annon, 2015-16).

Cowpea is a warm weather and drought resistant crop. grown for grain, vegetable and fodder purposes both in summer and kharif season. In India, it is mainly grown in Rajasthan, Gujarat, Maharashtra, Centre India and some regions of Southern India. In India, cowpea is cultivated over 3.9 million hectares with a production of 2.21 million tonnes with the national productivity of $683 \mathrm{~kg} / \mathrm{ha}$ (Singh and Lourduraj, 2014).

In Gujarat, cowpea occupies about 18,811 ha area with the production of 1, 66,391 MT (Anon., 2012). The exploitation of hybrid vigour as resource to increasing the yields of agricultural crops has become one of the most important technique in plant breeding. The heterosis expresses the superiority of $F_{1}$ hybrid over its parents in term of yield and other traits.

However, in autogamous crop like cowpea the possibility of its commercial exploitation is rather remote particularly because of flower biology and the practical difficulties involved in hybrid seed production.

However, information about heterosis and inbreeding depression for the identification of potential crosses which can offer maximum chances of isolating transgressive segregates is crucial in self pollinated crops. In the present study an attempt was made to estimate the extent of heterosis for seed yield and yield attributes in cowpea. In addition, inbreeding depression was also estimated for yield and yield attributes. The relative ranking of most heterotic crosses for different characters was quite different with change, indicating appreciable influence of environment in the expression of various traits.

\section{Materials and Methods}

The present investigation was carried out during kharif 2016, at College Farm, N. M. College of Agriculture, Navsari Agricultural University, Navsari located at $22^{\circ} 57^{\prime} \mathrm{N}$ latitude and $72^{\circ} 54^{\prime} \mathrm{E}$ longitudes at an altitude of $11.98 \mathrm{~m}$ above the mean sea level. The details of cowpea were used to study are given in Table 1.

Six generations components $\mathrm{P}_{1}, \mathrm{P}_{2}, \mathrm{~F}_{1}, \mathrm{~F}_{2}$, $\mathrm{BC}_{1}$ and $\mathrm{BC}_{2}$ of five crosses were involved eight diversified cultivars of cowpea. The $F_{1}$ hybrids were generated by crossing of above eight parents during Kharif 2014 (Table 2).

Selfing of $\mathrm{F}_{1} \mathrm{~s}$ was done in the same season (summer and kharif 2015) to get $\mathrm{F}_{2} \mathrm{~S}$. six generations $\left(\mathrm{P}_{1}, \mathrm{P}_{2}, \mathrm{~F}_{1}, \mathrm{~F}_{2}, \mathrm{BC}_{1}\right.$ and $\left.\mathrm{BC}_{2}\right)$ of each of the five crosses were sown during kharif-2016 in compact family block design with three replications.

Each replication was divided in five compact blocks. Each five crosses consisting of six generations were randomly allotted to the blocks. Six generations were than randomly allotted to each plot within a block. Each plot consisted of one row of parents and $F_{1} s$, two rows of the backcrosses and four rows of the $\mathrm{F}_{2}$ generations of each cross. Inter and intra row spacing was $45 \mathrm{~cm}$ and $10 \mathrm{~cm}$, respectively. Recommended agronomic practices in vogue along with necessary plant protection measures were timely adopted for successful rising of the good crop.

At the time of crossing, after the initiation of flower buds, the unopened, healthy buds were selected and emasculated between 4 to 6 p.m. with needle and forceps. While emasculating the flower buds, maximum care was taken to avoid injury either to any of the floral organs or flower stalk. 
Five plants from each of the $\mathrm{P}_{1}, \mathrm{P}_{2}, \mathrm{~F}_{1}, 20$ plants from $\mathrm{F}_{2}$ were randomly selected per replication and observations were recorded on single plant basis Harvest index (\%) was worked out from randomly selected five plants from each plot at the time of harvest and their average was recorded.

Seed yield per plant $(\mathrm{g})$
Harvesting index $(\%)=\frac{\text { P }}{\text { Biological yield per plant }(\mathrm{g})}$
Biolog

Protein content of dry seeds were determined by estimating the nitrogen content as per the method kjeldhal's method (Jackson, 1967) and multiplying the nitrogen content with a factor 6.25 and expressed on per cent basis for each genotypes.

Mean values obtained from the observations recorded on representative plants and samples for quantitative and biochemical characters for each entry in each family main and subplots were used for statistical computation.

\section{Results and Discussion}

The manifestation of heterosis, heterobeltiosis and inbreeding depression are presented in Table 3 and 4. The results revealed significant positive and negative mid parent and better parent's heterosis in many crosses for different characters studied. The high values for heterotic effects also indicated that the parents used for the study were widely diverse.

The highest heterosis of $44.72 \%$ heterobeltiosis of $18.94 \%$ and lowest inbreeding depression of $14.14 \%$ were exhibited by cross Waghi local x W-203-1. High and positive heterosis for seed yield in cowpea have been reported by several earlier worker, viz., Patel et al., (2009), Aremu and Adewale (2010), Rashwan (2010), Yadav et al., (2010), Adeyanju et al., (2012), Kajale et al., (2013), Patel et al., (2013) and Nautiyal et al., (2015).

In cowpea, pods per plant, seeds per pod and 100 seed weight are the three major yield components. Heterosis and inbreeding depression value for these three components along with seed yield of five heterotic crosses are given in Table 1 and Table 2. From these results it is apparent that Waghi local $\mathrm{x}$ W203-1 hybrid have potentiality for improving yield through adjustment of three vital yield components.

The results on inbreeding depression revealed that high inbreeding depression for seed yield was mainly due to the inbreeding depression for its components. Relationship between heterotic response and inbreeding depression (i.e. crosses showing high heterosis also show high inbreeding depression) suggests the importance of non- additive gene in cowpea. The results are in close agreement with those of Kheradanam et al., (1975) who also noticed that inbreeding depression in yield was due to inbreeding depression in yield attributing components in cowpea.

In case of days to $50 \%$ flowering only one cross Waghi local x W-203-1 depicted significant and negative relative heterosis and three crosses showed significant and positive relative heterosis. These results are in conformity with those obtained by Mehta et al., (2000), Viswanatha et al., (2006), Lal et al., (2007), Patel et al., (2009), Rashwan (2010), Adeyanju et al., (2012), Patel et al., (2013) and Nautiyal et al., (2015).

Only one cross Waghi local x W-203-1 showed negative and significant relative heterosis for days to maturity. Similar results were reported by Patel et al., (2009), Aremu and Adewale (2010), Adeyanju et al., (2012), Patel et al., (2013), Nautiyal et al., (2015). Panday and Singh (2015) and Pathak (2016). 
The magnitude of heterosis and heterobeltiosis were significantly high for number of branches per plant $(43.91 \%$ and $28.68 \%$ ) in cross Pant lobia-2 x GC-3 and Waghi local x W-203-1 followed by number of clusters per plant $(35.61 \%$ and $22.66 \%$ ) in cross Waghi local x W-203-1and Pant lobia1x BRDCP-11 and number of pods per plant (26.99\% and $16.94 \%)$ in cross Pant lobia-2 $x$ GC-3. The high values of heterosis for these three characters may be due to dominance or epistasis or both. Similar results have been observed by Joseph and Santhoshkumar (2000), Lal et al., (2007), Meena et al., (2009), Ojo et al., (2009), Patel et al., (2009), Adeyanju et al., (2012), Kajale et al., (2013), Patel et al., (2013), Nautiyal et al., (2015) and
Pandey and Singh (2015) and Pathak (2016).

The heterosis for number of seed per pod and pod length was moderate for cross Waghi local x W-203-1 and high for cross GC-3 xCDP-107. Positive heterosis for 100 seed weight was significant in three crosses. Thus the trait seems to be the control of dominance effect. For harvest index, all the crosses had positive and highly significant relative heterosis. All the crosses exhibited the positive and highly significant relative heterosis for protein content. Joseph and Santhosh kumar (2000), Pal et al., (2003), Lal et al., (2007), Patel et al., (2009), Patel et al., (2013), Nautiyal et al., (2015) and Pathak (2016) were observed similar results.

Table.1 Cowpea were used to study

\begin{tabular}{|c|c|c|c|c|c|c|c|c|c|}
\hline \multirow{2}{*}{$\begin{array}{l}\text { Sr. } \\
\text { no }\end{array}$} & \multirow{2}{*}{$\begin{array}{c}\text { Name of } \\
\text { Genotype }\end{array}$} & \multicolumn{8}{|c|}{ Character } \\
\hline & & $\begin{array}{c}\text { Plant } \\
\text { Growth Habit }\end{array}$ & $\begin{array}{l}\text { Plant } \\
\text { Habit }\end{array}$ & $\begin{array}{l}\text { Leaf } \\
\text { Size }\end{array}$ & $\begin{array}{c}\text { Seed } \\
\text { Colour }\end{array}$ & $\begin{array}{l}\text { Seeds per } \\
\text { pod }\end{array}$ & $\begin{array}{l}\text { Pod } \\
\text { length }\end{array}$ & $\begin{array}{l}\text { Seed } \\
\text { Size }\end{array}$ & $\begin{array}{l}\text { Disease } \\
\text { Reaction }\end{array}$ \\
\hline 1 & $\begin{array}{l}\text { Pant } \\
\text { Lobia -1 }\end{array}$ & Semi spreading & ID & Large & White & $10-16$ & Long & Large & MS \\
\hline 2 & $\begin{array}{l}\text { BRDCP- } \\
11\end{array}$ & Erect & ID & Large & White & $8-10$ & Short & Small & MR \\
\hline 3 & $\begin{array}{l}\text { Pant } \\
\text { Lobia -2 }\end{array}$ & Spreading & ID & Large & Red & $11-16$ & Long & Large & MS \\
\hline 4 & GC-3 & Erect & ID & $\begin{array}{l}\text { Mediu } \\
\mathrm{m}\end{array}$ & $\begin{array}{l}\text { Light } \\
\text { Cream }\end{array}$ & $8-10$ & Short & Small & MR \\
\hline 5 & $\begin{array}{l}\text { Waghi } \\
\text { Local }\end{array}$ & Semi spreading & ID & Large & $\begin{array}{l}\text { Light } \\
\text { Cream } \\
\text { Yellow }\end{array}$ & $10-12$ & Medium & Medium & MS \\
\hline 6 & W-203-1 & Semi spreading & ID & Large & $\begin{array}{l}\text { Light } \\
\text { Cream } \\
\text { Yellow }\end{array}$ & $9-13$ & Medium & Medium & MS \\
\hline 7 & KM-5 & Semi spreading & D & $\begin{array}{l}\text { Mediu } \\
\mathrm{m}\end{array}$ & Brown & $9-15$ & Long & Medium & MS \\
\hline 8 & CDP-107 & Erect & D & $\begin{array}{l}\text { Mediu } \\
\mathrm{m}\end{array}$ & $\begin{array}{l}\text { Light } \\
\text { Cream } \\
\text { Yellow }\end{array}$ & $10-13$ & Medium & Medium & MS \\
\hline
\end{tabular}


Table. 2 The $\mathrm{F}_{1}$ hybrids were developed

\begin{tabular}{|c|c|c|}
\hline Cross & Generation & Details \\
\hline I & \multicolumn{2}{|c|}{ Pant Lobia $-1 \times$ BRDCP-11 } \\
\hline & $\mathrm{P}_{1}$ & Pant Lobia -1 \\
\hline & $\mathrm{P}_{2}$ & BRDCP-11 \\
\hline & $\mathrm{F}_{1}$ & (Pant Lobia $-1 \times$ BRDCP-11) \\
\hline & $\mathrm{F}_{2}$ & (Pant Lobia $-1 \times$ BRDCP-11) $\mathrm{F}_{1}$ selfed \\
\hline & $\mathrm{BC}_{1}$ & $\begin{array}{l}\text { (Pant Lobia }-1 \times \text { BRDCP-11) x Pant } \\
\text { Lobia }-1\end{array}$ \\
\hline & $\mathrm{BC}_{2}$ & $\begin{array}{l}\text { (Pant Lobia }-1 \times \text { BRDCP-11) } \mathrm{x} \\
\text { BRDCP-11 }\end{array}$ \\
\hline \multirow[t]{7}{*}{ II } & \multicolumn{2}{|c|}{ Pant Lobia $-2 \times$ GC -3} \\
\hline & $\mathrm{P}_{1}$ & Pant Lobia -2 \\
\hline & $\mathrm{P}_{2}$ & $\mathrm{GC}-3$ \\
\hline & $\mathrm{F}_{1}$ & (Pant Lobia $-2 \times$ GC -3 ) \\
\hline & $\mathrm{F}_{2}$ & $($ Pant Lobia $-2 \times$ GC -3$) F_{1}$ selfed \\
\hline & $\mathrm{BC}_{1}$ & (Pant Lobia $-2 \times$ GC -3$) \times$ Pant Lobia -2 \\
\hline & $\mathrm{BC}_{2}$ & (Pant Lobia $-2 \times$ GC -3$) \times$ GC -3 \\
\hline \multirow[t]{7}{*}{ III } & \multicolumn{2}{|c|}{ Waghai Local $\times$ W-203-1 } \\
\hline & $\mathrm{P}_{1}$ & Waghai Local \\
\hline & $\mathrm{P}_{2}$ & W-203-1 \\
\hline & $\mathrm{F}_{1}$ & (Waghai Local × W-203-1) \\
\hline & $\mathrm{F}_{2}$ & (Waghai Local $\times$ W-203-1) $F_{1}$ selfed \\
\hline & $\mathrm{BC}_{1}$ & $\begin{array}{l}\text { (Waghai Local × W-203-1) x Waghai } \\
\text { Local }\end{array}$ \\
\hline & $\mathrm{BC}_{2}$ & (Waghai Local $\times$ W-203-1) x W-203-1 \\
\hline \multirow[t]{7}{*}{ IV } & \multicolumn{2}{|c|}{$\mathrm{KM}-5 \times \mathrm{GC}-3$} \\
\hline & $\mathrm{P}_{1}$ & KM-5 \\
\hline & $\mathrm{P}_{2}$ & GC-3 \\
\hline & $\mathrm{F}_{1}$ & $(\mathrm{KM}-5 \times \mathrm{GC}-3)$ \\
\hline & $\mathrm{F}_{2}$ & $(\mathrm{KM}-5 \times \mathrm{GC}-3) \mathrm{F}_{1}$ selfed \\
\hline & $\mathrm{BC}_{1}$ & $(\mathrm{KM}-5 \times \mathrm{GC}-3) \times \mathrm{KM}-5$ \\
\hline & $\mathrm{BC}_{2}$ & $(\mathrm{KM}-5 \times \mathrm{GC}-3) \times \mathrm{GC}-3$ \\
\hline \multirow[t]{7}{*}{$\mathbf{V}$} & \multicolumn{2}{|c|}{ GC-3 x CDP-107 } \\
\hline & $\mathrm{P}_{1}$ & GC-3 \\
\hline & $\mathrm{P}_{2}$ & CDP-107 \\
\hline & $\mathrm{F}_{1}$ & $(\mathrm{GC}-3 \times \mathrm{CDP}-107)$ \\
\hline & $\mathrm{F}_{2}$ & $(\mathrm{GC}-3 \times \mathrm{CDP}-107) \mathrm{F}_{1}$ selfed \\
\hline & $\mathrm{BC}_{1}$ & $(\mathrm{GC}-3 \times \mathrm{CDP}-107) \times \mathrm{GC}-3$ \\
\hline & $\mathrm{BC}_{2}$ & $(\mathrm{GC}-3 \times \mathrm{CDP}-107) \times \mathrm{CDP}-107$ \\
\hline
\end{tabular}


Table.3 Estimates of relative heterosis (R.H \%), hetero beltiosis (H.B \%) and inbreeding depression (I.D \%) for days to $50 \%$ flowering, days to maturity, plant height $(\mathrm{cm})$, number of branches per plant, number of clusters per plant, number of pods per plant, number of seeds per pod and pod length in five crosses of cowpea

\begin{tabular}{|c|c|c|c|c|c|c|}
\hline \multicolumn{4}{|c|}{ Days to $50 \%$ flowering, } & \multicolumn{3}{|c|}{ Days to maturity } \\
\hline Crosses & Heterosis (\%) over MP & Heterosis (\%) over BP & ID $(\%)$ & Heterosis (\%) over MP & Heterosis (\%) over BP & ID $(\%)$ \\
\hline Cross - 1 & $5.76 * * \pm 0.49$ & $8.44 * * \pm 0.61$ & $6.53 * * \pm 0.79$ & $6.80 * * \pm 0.51$ & $2.92 * * \pm 0.80$ & $8.23^{* *} \pm 0.76$ \\
\hline Cross - 2 & $0.82 \pm 0.56$ & $5.20 * * \pm 0.65$ & $-3.65 \pm 1.02$ & $8.46^{* *} \pm 0.65$ & $-4.68^{* *} \pm 0.71$ & $8.66^{* *} \pm 1.00$ \\
\hline Cross - 3 & $-10.67 * * \pm 0.55$ & $-8.79 * * \pm 0.66$ & $-19.67 * * \pm 0.77$ & $-5.13 * * \pm 0.96$ & $-7.49 * * \pm 1.08$ & $-10.83 * * \pm 1.07$ \\
\hline Cross - 4 & $10.44 * * \pm 0.41$ & $13.66 * * \pm 0.53$ & $15.35^{* *} \pm 0.74$ & $10.36^{* *} \pm 0.50$ & $5.58^{* *} \pm 0.66$ & $2.24 * \pm 0.86$ \\
\hline Cross - 5 & $13.30 * * \pm 0.48$ & $17.78 * * \pm 0.60$ & $13.90 * \pm 0.69$ & $6.28 * * \pm 0.65$ & $4.19 * * \pm 0.72$ & $8.84 * * \pm 0.84$ \\
\hline \multicolumn{4}{|c|}{ Plant height (cm) } & \multicolumn{3}{|c|}{ Number of branches per plant } \\
\hline Cross - 1 & $-11.84 * * \pm 1.11$ & $-24.25 * * \pm 1.18$ & $-10.84^{* *} \pm 1.50$ & $25.40^{* *} \pm 0.47$ & $22.27^{*} \pm 0.59$ & $17.02 * \pm 0.57$ \\
\hline Cross - 2 & $-13.16 * * \pm 0.81$ & $-34.82 * * \pm 0.91$ & $-8.43 * * \pm 1.84$ & $43.91^{* *} \pm 0.32$ & $20.49^{* *} \pm 0.37$ & $24.38 * * \pm 0.47$ \\
\hline Cross - 3 & $1.84 \pm 1.11$ & $-3.48^{*} \pm 1.29$ & $-10.50 * * \pm 1.60$ & $31.58^{* *} \pm 0.36$ & $28.68^{* *} \pm 0.39$ & $29.29 * * \pm 0.54$ \\
\hline Cross - 4 & $30.71 * * \pm 1.44$ & $9.60 * * \pm 1.48$ & $4.17 \pm 1.92$ & $15.12 * * \pm 0.27$ & $-5.71 \pm 0.32$ & $-6.31 \pm 0.30$ \\
\hline Cross - 5 & $13.26 * * \pm 0.64$ & $6.90 * * \pm 0.67$ & $11.38^{* *} \pm 0.89$ & $-7.26 \pm 0.38$ & $-19.86^{* *} \pm 0.40$ & $-5.12 \pm 0.46$ \\
\hline \multicolumn{4}{|c|}{ Number of clusters per plant } & \multicolumn{3}{|c|}{ Number of pods per plant } \\
\hline Cross - 1 & $29.22 * * \pm 0.51$ & $22.66^{* *} \pm 0.67$ & $11.78 * * \pm 0.55$ & $20.54^{* *} \pm 0.51$ & $11.29 * * \pm 0.59$ & $11.90 * * \pm 0.72$ \\
\hline Cross - 2 & $35.61 * * \pm 0.37$ & $19.64 * * \pm 0.42$ & $26.68 * * \pm 0.36$ & $26.99 * * \pm 0.30$ & $16.94 * * \pm 0.35$ & $28.17 * * \pm 0.76$ \\
\hline Cross - 3 & $26.64 * * \pm 0.37$ & $19.61^{* *} \pm 0.41$ & $19.81 * * \pm 0.48$ & $24.59^{* *} \pm 0.66$ & $15.35^{* *} \pm 0.82$ & $26.16^{* *} \pm 0.94$ \\
\hline Cross - 4 & $0.48 \pm 0.29$ & $-12.93 * * \pm 0.28$ & $-16.45^{* *} \pm 0.36$ & $20.21^{* *} \pm 0.38$ & $13.87^{* *} \pm 0.44$ & $21.62 * * \pm 0.71$ \\
\hline Cross - 5 & $20.44 * * \pm 0.30$ & $15.39 * * \pm 0.35$ & $-0.31 \pm 0.42$ & $18.74 * * \pm 0.66$ & $6.94 * \pm 0.64$ & $7.85 \pm 0.97$ \\
\hline \multicolumn{4}{|c|}{ Number of seeds per pod } & \multicolumn{3}{|c|}{ Pod length } \\
\hline Cross - 1 & $0.96 \pm 0.56$ & $-12.29 * \pm 0.57$ & $13.22 * \pm 0.56$ & $3.84 \pm 0.51$ & $-9.83^{* *} \pm 0.50$ & $12.17 * * \pm 0.57$ \\
\hline Cross - 2 & $0.11 \pm 0.30$ & $-11.75^{* *} \pm 0.34$ & $4.02 \pm 0.73$ & $1.78 \pm 0.37$ & $-12.28 * * \pm 0.40$ & $2.75 \pm 0.66$ \\
\hline Cross - 3 & $13.65^{* *} \pm 0.41$ & $5.15 \pm 0.50$ & $-3.43 \pm 0.56$ & $19.42^{* *} \pm 0.32$ & $9.26^{* *} \pm 0.37$ & $-5.85 \pm 0.71$ \\
\hline Cross - 4 & $-4.57 \pm 0.41$ & $-19.71 * * 0.50$ & $2.25 \pm 0.50$ & $3.06 \pm 0.42$ & $-11.40 * * \pm 0.56$ & $3.84 \pm 0.69$ \\
\hline Cross - 5 & $42.23 * * \pm 0.42$ & $28.21 * * \pm 0.55$ & $37.54 * * \pm 0.61$ & $42.41 * * \pm 0.55$ & $28.35 * * \pm 0.64$ & $35.30 * * \pm 0.84$ \\
\hline
\end{tabular}


Table.4 Estimates of relative heterosis (R.H \%), Hetero Beltiosis ( H.B \%) and Inbreeding Depression (I.D \%) for 100 seed weight (gm), seed yield per plant (gm), harvest index (\%), protein content (\%), Chlorophyll contents and leaf area $\left(\mathrm{cm}^{2}\right)$ in five crosses of cowpea

\begin{tabular}{|c|c|c|c|c|c|c|}
\hline \multicolumn{4}{|c|}{100 seed weight (gm) } & \multicolumn{3}{|c|}{ Seed yield per plant (gm) } \\
\hline Crosses & Heterosis (\%) over MP & Heterosis (\%) over BP & ID $(\%)$ & Heterosis (\%) over MP & Heterosis (\%) over BP & ID $(\%)$ \\
\hline Cross - 1 & $3.35 \pm 0.36$ & $-10.54 * * \pm 0.43$ & $-2.57 \pm 0.65$ & $17.93 * * \pm 0.56$ & $-1.35 \pm 0.66$ & $17.74 * * \pm 0.75$ \\
\hline Cross - 2 & $19.34 * * \pm 0.34$ & $14.76^{* *} \pm 0.33$ & $-1.17 \pm 0.62$ & $26.73^{* *} \pm 0.46$ & $14.56^{* *} \pm 0.63$ & $21.65^{* *} \pm 1.26$ \\
\hline Cross - 3 & $25.07 * * \pm 0.41$ & $17.93 * * \pm 0.44$ & $10.14 \pm 0.49$ & $44.72 * * \pm 0.98$ & $18.94 * * \pm 1.09$ & $14.14 * * \pm 1.61$ \\
\hline Cross - 4 & $23.92 * * \pm 0.39$ & $19.72 * * \pm 0.50$ & $15.81 * * \pm 0.49$ & $31.10^{* *} \pm 1.03$ & $15.86^{* *} \pm 1.12$ & $18.65 * * \pm 1.34$ \\
\hline Cross - 5 & $-6.97 \pm 0.45$ & $-9.09 \pm 0.52$ & $-19.73 * * \pm 0.60$ & $13.75 * * \pm 1.19$ & $5.17 \pm 1.01$ & $17.15^{* *} \pm 1.52$ \\
\hline \multicolumn{4}{|c|}{ Harvest index (\%) } & \multicolumn{3}{|c|}{ Protein content $(\%)$} \\
\hline Cross - 1 & $7.60 * * \pm 0.87$ & $-5.16^{* *} \pm 0.91$ & $15.70 * * \pm 1.59$ & $19.90 * * \pm 0.51$ & $15.58 * * \pm 0.58$ & $19.60 * \pm 0.52$ \\
\hline Cross - 2 & $15.79 * * \pm 1.08$ & $13.85^{* *} \pm 1.13$ & $7.56 \pm 1.27$ & $7.90 * * \pm 0.41$ & $5.10 * * \pm 0.42$ & $12.29 \pm 0.54$ \\
\hline Cross - 3 & $21.15^{* *} \pm 1.15$ & $7.68 * * \pm 1.39$ & $8.11^{* *} \pm 1.24$ & $5.87 * * \pm 0.20$ & $2.66^{*} \pm 0.24$ & $8.75^{* *} \pm 0.21$ \\
\hline Cross - 4 & $20.13 * * \pm 1.00$ & $17.09 * * \pm 1.05$ & $6.80 * * \pm 1.23$ & $10.14 * * \pm 1.02$ & $5.04 \pm 0.58$ & $17.32 \pm 0.57$ \\
\hline Cross - 5 & $11.64 * * \pm 0.82$ & $8.87 * * \pm 0.78$ & $6.19 \pm 0.91$ & $4.33 * * \pm 0.27$ & $0.61 \pm 0.37$ & $13.06 * * \pm 0.43$ \\
\hline \multicolumn{4}{|c|}{ Chlorophyll Contents } & \multicolumn{3}{|c|}{ Leaf area } \\
\hline Cross - 1 & $6.56 * * \pm 0.71$ & $2.50 \pm 0.84$ & $5.26^{*} \pm 0.96$ & $8.02 * * \pm 0.77$ & $5.26 \pm 0.94$ & $6.02 * \pm 0.84$ \\
\hline Cross - 2 & $9.92 * * \pm 0.72$ & $8.01 * * \pm 0.84$ & $5.18 * \pm 0.95$ & $11.26^{* *} \pm 0.64$ & $4.91 * * \pm 0.62$ & $16.99 * * \pm 1.00$ \\
\hline Cross - 3 & $11.10 * * \pm 1.32$ & $5.88 \pm 1.47$ & $7.84^{*} \pm 1.53$ & $16.35 * * \pm 1.19$ & $4.61 \pm 1.28$ & $4.59 \pm 1.44$ \\
\hline Cross - 4 & $8.92 * * \pm 0.49$ & $3.21 * \pm 0.55$ & $8.17 * * \pm 1.05$ & $0.56 \pm 0.74$ & $-6.31 * * \pm 0.74$ & $-3.93 \pm 1.14$ \\
\hline Cross - 5 & $11.09 * * \pm 0.71$ & $6.45^{* *} \pm 0.66$ & $4.66 \pm 1.49$ & $-2.35 \pm 1.04$ & $-10.60 * * \pm 1.12$ & $1.17 \pm 0.99$ \\
\hline
\end{tabular}


The heterosis for chlorophyll contents all the crosses exhibited the positive and highly significant whereas, only three crosses exhibited the positive and highly significant heterobeltiosis for this trait.

All the five $F_{2}$ crosses exhibited significant inbreeding depression in respect of seed yield per plant. The results are in conformity with the finding of Kheradanam et al., (1975). Joseph and Santhosh kumar (2000), Pal et al., (2003), Lal et al., (2007), Patel et al., (2009), Adeyanju et al., (2012), Kajale et al., (2013), Patel et al., (2013) and Nautiyal et al., (2015).

On reviewing the results of heterosis and per se performance the hybrids having more than $15 \%$ heterosis over better parent viz.; Waghi local x W-203-1 and KM-5 x GC-3 for yield and yield contributing traits can safely be utilized for improving yield through exploiting hybrid vigour. Singh (1983) advocated utilizing hybrids having more than $15 \%$ heterosis over better parent for improving cowpea. However, considering the cleistogamous flower, self-pollinated nature and absence of commercially exploitable male sterility system in cowpea, heterosis per se may be of limited value. The cross, Waghi local $x$ W-203-1 showing high per se performance, high heterobeltiosis and less inbreeding for seed yield can be safely utilized for improvement of seed yield in cowpea through selection in advance generation

\section{References}

Adeyanju, A. O., Ishiyaku, M. F., Echekwu, C. A. and Olarewaju, J. D. (2012). Generation mean analysis of dual purpose traits in cowpea [Vigna unguiculata (L.) Walp]. African J. of Biot., 11 (46): 10473-10483.

Anonymous, (2012). Director of Horticulture, Gandhinagar, Gujarat.
Anonymous, (2015-16). www.indiaagristat.com/table/agriculture /total pulses.

Aremu, C. O. and Adewale, B. D. (2010). Heterosis and phenolic performance in a selected cross of cowpea [Vigna unguiculata (L.) Walp]. For humid environment performance. Agric. J., 5 (5): 292-296.

Jackson, M. L. (1967). Soil chemical analysis, Prentice Hall Int., New Delhi.

Joseph, J. and Santoshkumar, A. V. (2000). Genetic analysis of metric traits in green gram (Vigna radiata (L.) Wilczek). International J. Tropical Agric., 18 (2):133-139.

Kajale, D. B., Ravindrababu, Y., Prajapati, D. B. and Khule, A. A. (2013). Heterosis in cowpea (Vigna unguiculata L. Walp). Veg. Sci.,40 (2): 237-239.

Kheradanam, M. , Bassiri, A. and Nikhejad, M. (1975). Heterosis, inbreeding depression and reciprocal effects for yield and some yield components in a cowpea cross. Crop. sci., 15 (5): 689691.

Lal, H., Singh, A. P., Verma, A., Rai, M., Singh, S. N., Nath, V. and Ram, D. (2007). Heterosis and inbreeding depression in cowpea (Vigna unguiculata (L.) Walp.). Veg. Sci., 34 (2): 557-563.

Meena, R., Pithia, M. S., Savaliya, J. J. and Pansuria, A. G. (2009). Heterosis in vegetable cowpea (Vigna unguiculata (L.) Walp.). Crop improvement, 36 (1) : 1- 5

Mehta, D. R. (2000). Comparison of observed and expected heterosis and inbreeding depression in four cowpea crosses. Indian J. Agril. Rrs.. 34 (2): 97-101.

Nautiyal, M. K., Upreti, M., Kousar, K. N. and Shrotria, P. K. (2015). Estimation of gene effects, heterosis and inbreeding depression for yield contributing traits in cowpea. Environment and Ecology. 
32 (3): 975-979.

Ojo, D. K., Oduwaye, O. A., Idehen,E.O., Adekoya, M. A. and Akinsip, K.T. (2009). Inheritance of seed Yield and yield related characters in cowpea (Vigna unguiculata (L.) Walp). Journal of Agriculture, Biotechnology\& Ecology, 2: 18-23.

Pal, A. K., Singh, B. and Maurya, A. N. (2003).Inbreeding depression in cowpea (Vigna unguiculata (L.) Walp.). J. of Applied Hort., 5 (2): 105-107.

Pandey, B. and Singh, Y.V. (2015). Heterosis for yield and yield contributing characters in cowpea genotypes. Legume Research, 38 (5): 570-574.

Patel, H., Patel, J. B., Sharma, S. C. and Acharya, S. (2013). Heterosis and inbreeding depression study in cowpea [Vigna unguiculata (L.) Walp.]. An International e-Journal. 2 (2): 165-172.

Patel, S. J., Desai, R.T., Bhakta, R.S., Patel, D.U., Kodappully, V.C. and Mali, S.C. (2009). Heterosis studies in cowpea [vigna unguiculata (L.) Walp]. Legume Res., 32 (3): 199-202.

Pathak, A. R. (2016). Generation mean analysis for yield and yield components in cowpea [Vigna unguiculata (L.)Walp.]. Thesis, Navsari agricultural University, Navasari.

Rashwan, A. M. A. (2010). Estimation of some genetic parameters using six populations of two cowpea hybrids. Asian J. of Crop Sci. 2: 261-267.

Singh, R. P. (1983). Heterosis in cowpea. $J$. Res. Asam Agric.Unic., 4 (1): 12-14.

Singh, R. S. R. and Lourduraj, A. C. (2014). Growth and yield of cowpea as influenced by integrated nutrient management practices in preceding maize. Advance Research. Journal of Crop Improvement. 5 (1): 29-33.

Viswanatha, K. P., Bhushana, H. O., Yogeesh, L. N. and K. Devaraju (2006). Heterosis studies for yield and yield attributing traits in cowpea [Vigna unguiculata (L.) Walp.]. Forage Res., 32 (3): 148-151.

Yadav, K. S., Yadava, H.S. and Dixit, H. (2010). Heterosis and inbreeding depression in cowpea. International $J$. of Agric. Sci., 6 (2): 537-540.

\section{How to cite this article:}

Gupta, R.P., S. R. Patel, Dinisha Abhishek, S.S. Patil and Patel, H.N. 2020. Heterosis and Inbreeding Depression for Seed Yield Attributing Traits and Quality Parameter in Cowpea (Vigna unguiculata (L.) Walp.). Int.J.Curr.Microbiol.App.Sci. 9(08): 3335-3343. doi: https://doi.org/10.20546/ijcmas.2020.908.384 